\title{
POINT SCORE SYSTEMS AND FOOTBALL COACHING SECRECY
}

\author{
KJETIL K. HAUGEN
}

\begin{abstract}
In this paper, a game between two football (soccer) teams is analysed. The focus is on how the choice of point score system may affect Nash equilibria in a given simultaneous game and a corresponding sequential version. The reason for this choice, is (to some extent) experience related to the growing secrecy on pre-game strategic choices among football coaches.

It is demonstrated by the relatively simple game theory, that the point score system plays a vital role in how teams (coaches) will "play" such games, given that they are rational and recognize Nash equilibrium as a reasonable game prediction. In fact, some evidence on an increased tendency for more pre-game strategic secrecy is logically established in a move from a 2-1-0 point score system to a 3-1-0 point score system.
\end{abstract}

\section{INTRODUCTION}

Today's football (soccer) seems to include a lot more secrecy related to pre-game strategic choices. Teams forbid their players to talk to media and close their training grounds - presumably to keep certain strategic choices a secret from their opponents. Personally, I have limited scientific information stating that this has been a change from before, but I have a strong feeling that there has been a development in such a direction. At least, I can remember that announcing the players on a team well ahead of the match-day was quite common 20 to 30 years ago.

The question I will discuss in this paper is related to such a (possible) development. What has driven this increased tendency for secrecy. Is it the importance of sports? Has the amount of money at stake reached such heights that secrecy is necessary, or could there be alternative explanations? Here, a possibly unexpected dimension is examined - the point score system. Could the change from the original 2-1-0 (2 points for a win and one for a draw) to the 3-1-0 system have had any impact on the value of secrecy?

Increased secrecy comes of course at a cost. Keeping the information wanted by the media off this media is never free. The amounts can surely be discussed although not here. The point is simple. If secrecy is unnecessary, do not bother. In a Pareto setting, if the rules and regulations stimulate secrecy, perhaps one

$M S C$ (2010): primary 91; secondary 91A05,91A10, 91A 80.

Keywords: sequential game, simultaneous game, coaching, Nash equilibrium invariance.

This research has received no special funding. 
should change the rules instead of spending unnecessary resources on trying to keep such secrets.

In order to analyse this question, simple game theory is applied. It seems reasonable to compare a simultaneous game with a sequential version of the same game, given pay-off functions involving point score. If the point score system itself can be shown to impact Nash equilibria of such games - for instance in such a way that a tendency to "play" the simultaneous game differently than the sequential analogue - this ought to be both valuable as well as genuinely interesting information for the "football family".

The basic analytic approach is hence to open up for a coach to announce his secret (a sequential game) or keep it (a simultaneous game). A comparison of the Nash equilibrium outcomes of these two game types then opens up for arguments related to the value - or to be more specific - the absence of value of keeping the secret.

The paper is organized as follows. Section 2 discusses the relevant literature. Section 3 introduces the game framework and makes some necessary reformulations of the basic underlying framework, captured from [9]. The main analysis is performed in Sections 4 and 5, while Section 6 concludes and points out some additional research directions.

\section{Literature}

Several authors have discussed point score systems and their consequences in sport. Of direct relevance for this article (and football) is perhaps $[8,9]$ as well as $[5,7]$ and [12]. Other branches of sports activity and the role of the point score system has also been investigated - see for instance [3]. The above papers range from empiric studies via probabilistic to game models.

The methods which are used in this paper - comparing simultaneous games with sequential analogues - are of course well treated in general economic theory. In those branches of theory, the subjects of comparison are often referred to as Nash versus Stackelberg equilibrium. Some examples of work of relevance within this subject area are $[1,2,6,13]$ and [14].

\section{Game modelling}

\subsection{Basic assumptions}

The following basic assumptions are made:

1) The modelling framework of [9] (also applied in $[8,10,11]$ ) is adapted:

1.1) 2 teams $\left(T_{1}\right.$ and $\left.T_{2}\right)$ are engaged in an upcoming match. Each team chooses among two possible strategies $\left(O\right.$ and $\left.D^{1}\right)$;

1.2) The teams behave rationally, and are assumed to maximise the expected point scores;

\footnotetext{
${ }^{1}$ Although it is easy to read $O$ as offensive and $D$ as defensive, this is not necessarily the setting here. The strategies could for instance be two different team picks or two other strategies related to how the teams choose to play.
} 
1.3) Possible outcomes of the match are assumed stochastic (contingent on strategic choices) with probabilistic definitions

$$
\begin{gathered}
p_{i j}^{x y}=\operatorname{Pr}\left(T_{i} \text { beats } T_{j} \mid T_{i}=x \text { and } T_{j}=y\right), \\
p_{D}^{x y}=\operatorname{Pr}\left(\text { of a draw } \mid T_{i}=x \text { and } T_{j}=y\right), \\
x, y \in\{O, D\} \text { and } i, j \in\{1,2\} .
\end{gathered}
$$

2) A general point score system is used for analytic purposes. The system is defined by: A win for one of the teams generates $\omega$ points, a draw $\delta$ points and a loss (without loss of generality) 0 points. $\omega>\delta>0$.

3) The players of the game, teams $T_{1}$ and $T_{2}$, are assumed to have a choice of announcing their chosen strategy $(O$ or $D)$ before the game is played (a sequential option) or "keeping silent" (a simultaneous option). There is no possible disagreement on all the above facts. Hence, it is a game of complete information, but either of perfect or imperfect information depending on the sequential/simultaneous choice.

\subsection{Reformulations}

The reformulations $^{2}$ in [9] are applied

$$
\begin{aligned}
& p_{12}^{O O}=p_{21}^{O O}=p, \\
& p_{12}^{O D}=p_{21}^{D O}=s, \\
& p_{21}^{O D}=p_{12}^{D O}=r, \\
& p_{12}^{D D}=p_{21}^{D D}=q .
\end{aligned}
$$

Expected point scores (E(pay-off $T_{1}$ and $\left.T_{2}\right)$ ) can then be computed as shown in Table 1.

\begin{tabular}{cc|ccc|c|c}
$T_{1}$ & $T_{2}$ & $p_{12}$ & $p_{21}$ & $p_{D}$ & $\mathrm{E}\left(\right.$ Pay-off $\left.T_{1}\right)$ & $\mathrm{E}\left(\right.$ Pay-off $\left.T_{2}\right)$ \\
\hline $\mathrm{O}$ & $\mathrm{O}$ & $p$ & $p$ & $1-2 p$ & $\omega p+\delta(1-2 p)$ & $\omega p+\delta(1-2 p)$ \\
$\mathrm{D}$ & $\mathrm{O}$ & $r$ & $s$ & $1-r-s$ & $\omega r+\delta(1-r-s)$ & $\omega s+\delta(1-r-s)$ \\
$\mathrm{O}$ & $\mathrm{D}$ & $s$ & $r$ & $1-r-s$ & $\omega s+\delta(1-r-s)$ & $\omega r+\delta(1-r-s)$ \\
$\mathrm{D}$ & $\mathrm{D}$ & $q$ & $q$ & $1-2 q$ & $\omega q+\delta(1-2 q)$ & $\omega q+\delta(1-2 q)$
\end{tabular}

Table 1. Expected point scores for both teams

Now, some new variables are defined

$$
\begin{aligned}
\mu & =\omega p+\delta(1-2 p), \\
\gamma & =\omega q+\delta(1-2 q), \\
\theta & =\omega r+\delta(1-r-s) .
\end{aligned}
$$

Based on the definitions (3.5), (3.6) and (3.7) the normal form game as shown in Figure 1 appears:

\footnotetext{
${ }^{2}$ These equations (3.1), (3.2), (3.3) and (3.4) are derived based on an (initial) assumption of equally good teams.
} 


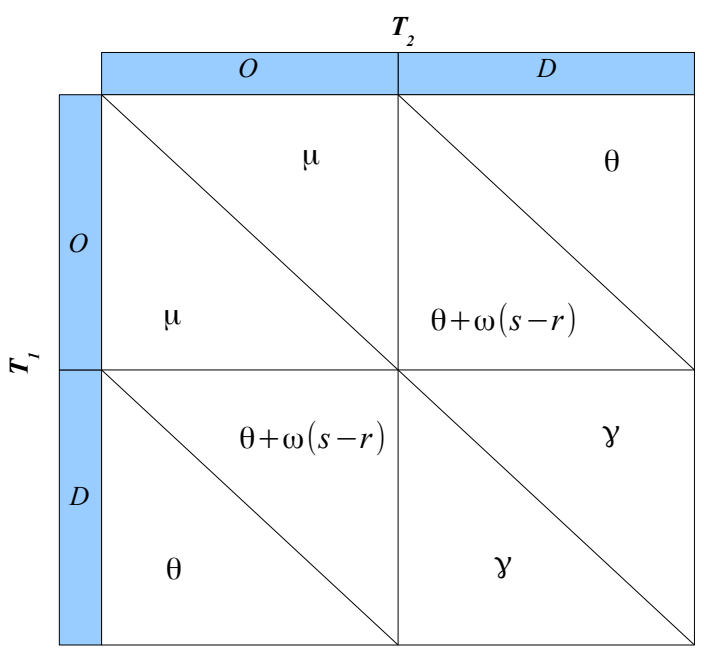

Figure 1. Two player simultaneous (normal form) game.

\section{Point SCORE System AND NASh EQUilibria}

Let me make a small digression before the game in Figure 1 is analysed further. By (3.5) and (3.6), $\mu-\gamma$ is computed as

$$
\begin{aligned}
\mu-\gamma & =\omega p+\delta(1-2 p)-(\omega q+\delta(1-2 q)) \\
& =\omega(p-q)-2 \delta(p-q)=(\omega-2 \delta)[p-q]
\end{aligned}
$$

The expression (4.1) is interesting. As can be readily observed, $\mu-\gamma=0$ or $(\mu=\gamma)$ is obtained either if $p=q$ or if $\omega=2 \delta$. The first condition $(p=q)$ is related to team performance and implies a situation where the team is equally good in winning or drawing a match, and is not so relevant here. However, the second condition, $\omega=2 \delta$, is far more interesting. The game in Figure 1 explains why. As can be observed in Figure 2, two inequalities define all best reply functions - observe red arrows, ellipses and rectangles.

That is, all Nash equilibria of the game are solely determined by

$$
\begin{aligned}
& \mu \gtreqless \theta, \\
& \gamma \gtreqless \theta+\omega(s-r) .
\end{aligned}
$$

Now, if (as discussed above) $\mu=\gamma$, inequalities (4.2) and (4.3) change to

$$
\begin{aligned}
& \mu \gtreqless \theta, \\
& \mu \gtreqless \theta+\omega(s-r) .
\end{aligned}
$$

Hence, if $s>r \Rightarrow \omega(s-r)>0$ and if (4.5) holds, so does (4.4). Alternatively, $s<r \Rightarrow \omega(s-r)<0$ and if (4.4) holds, so does (4.5). This argument holds for any direction of the inequality signs in (4.4), (4.5). To sum up: the Nash equilibria of the original game are defined by two (essentially) independent inequalities; (4.2) and (4.3), while a constraint $\mu=\gamma$ simplifies (the game) significantly into (in essence) a single inequality - either (4.4) or (4.5) depending on the sign of $s-r$. 


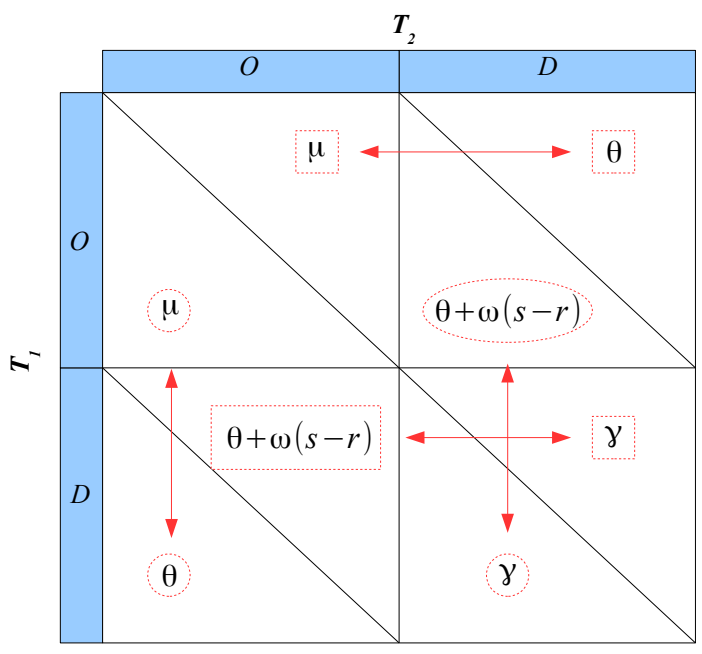

Figure 2. Best reply functions - ellipses (and circles) for $T_{1}$, rectangles (and squares) for $T_{2}$.

What is the reason for such a simplification? One reason is (as observed above), the constraint $\omega=2 \delta$. Today's point score system (3-1-0) does not satisfy this constraint. However, the previous (2-1-0) system did. This, in my opinion, is quite interesting. The fact that the choice of point score system has such significant influence on the analysis of these games is both surprising and relevant. One might ask if sport officials had any idea of such consequences when the 3-1-0 point score system was initiated.

\section{Point SCORE SYSTEMS AND SECRECY}

As discussed in the introduction, the hypothesis is related to how coaching secrecy variability is (or is not) linked up with the point score system and possible changes in this system.

Or, more directly formulated: Will changes in the point score system lead to possible strategic effects on football coaches' preferences related to keeping the team pick (or other pre-game sustainable strategic decisions) a secret?

However, before I move to this question, a few general game theoretic remarks are made. Firstly, observe in Figure 1 that the game at hand is symmetric. (Refer, for instance, to [6] for a thorough treatment of the concept of symmetry in games.)

Furthermore, to simplify the forthcoming analysis, the following theorem comes in handy:

Theorem 5.1. In $a 2$ by 2 symmetric simultaneous game, the order of follower/leader in the corresponding sequential game does not matter ${ }^{3}$.

A proof of the theorem is given in appendix A. In the forthcoming sections, this theorem is used to avoid comparing solutions of the simultaneous game with

\footnotetext{
${ }^{3}$ That is, the sequence of the players produces the same Nash equilibrium in both sequential versions for any pay-offs as long as the original simultaneous game is symmetric.
} 
two separate sequential games. As long as the game is symmetric, it is sufficient to investigate Nash equilibria in a single version of the sequential game.

Now, the following assumptions are made

$$
\mu>\theta \text { and } \gamma>\theta+\omega(s-r) .
$$

Given the assumptions (5.1) and inequalities (4.2) and (4.3), it is easy to see that the original simultaneous game is a "Stag-Hunt" game with two pure Nash equilibria and an (invisible) one in mixed strategies, see Figure 3.

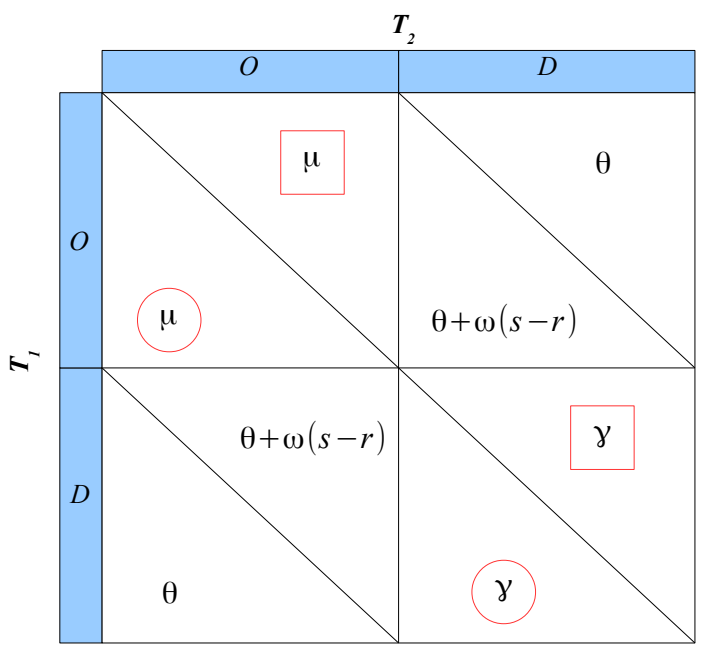

Figure 3. The "Stag-Hunt" outcome in the simultaneous game.

Furthermore, a (single) sequential version of the simultaneous game in Figure 1 is formulated, and shown in Figure 4.

By the assumptions (5.1), it is straightforward to find the best reply functions (red lines in Figure 5) for $T_{2}$ at the initial stage in Zermelo's algorithm, see Figure 5. Based on this information, it is readily observed that the only possible way the Nash equilibria in the original simultaneous game (Figure 1) can be replicated in the sequential game (Figure 4 ) is if

$$
\mu=\gamma
$$

As shown already (in equation (4.1)), this can be achieved if $\omega=2 \delta$. As a consequence, the strong link between the coaching secrecy and the point score design is established. If (in this example governed by the assumptions (5.1)) $\omega$ equals $2 \delta$, there is no difference between playing a sequential or a simultaneous game. This means that there is neither a potential gain, nor a potential loss (strategically) involved in announcing your pre-game strategy. Hence, if other arguments lead to a prefer-ability in strategy announcement, such a strategy may actually be beneficial, see Section 1. That is, the old point score regime (2-1-0) may have quite a different impact on the pre-game strategic coaching decisions compared with the new point score (3-1-0) regime. 


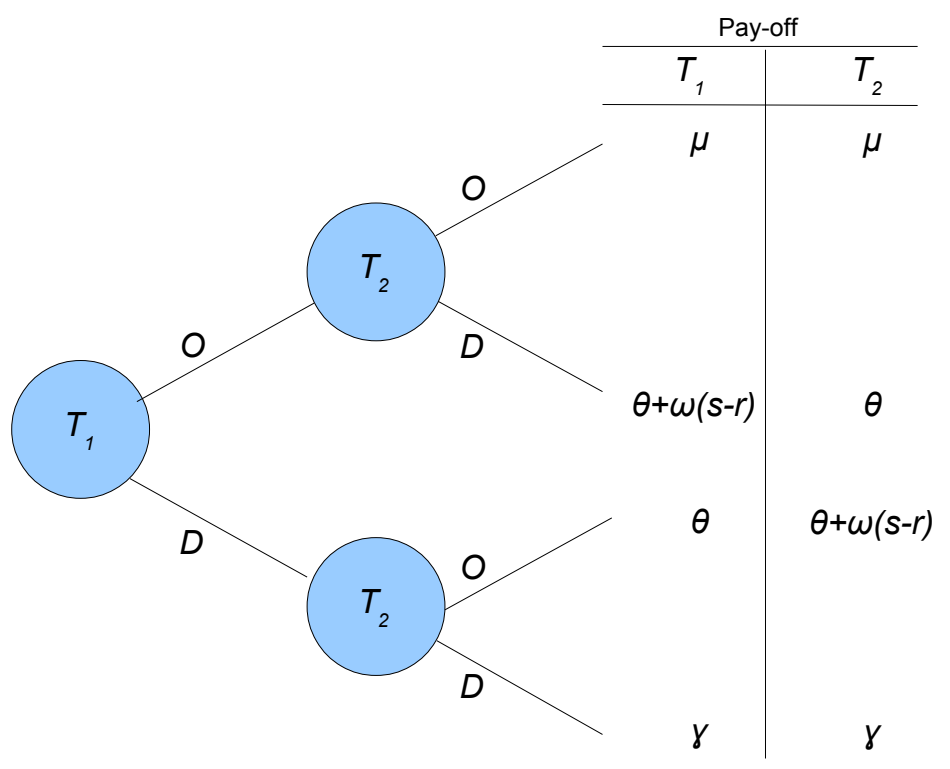

Figure 4. A sequential version of the original simultaneous game.

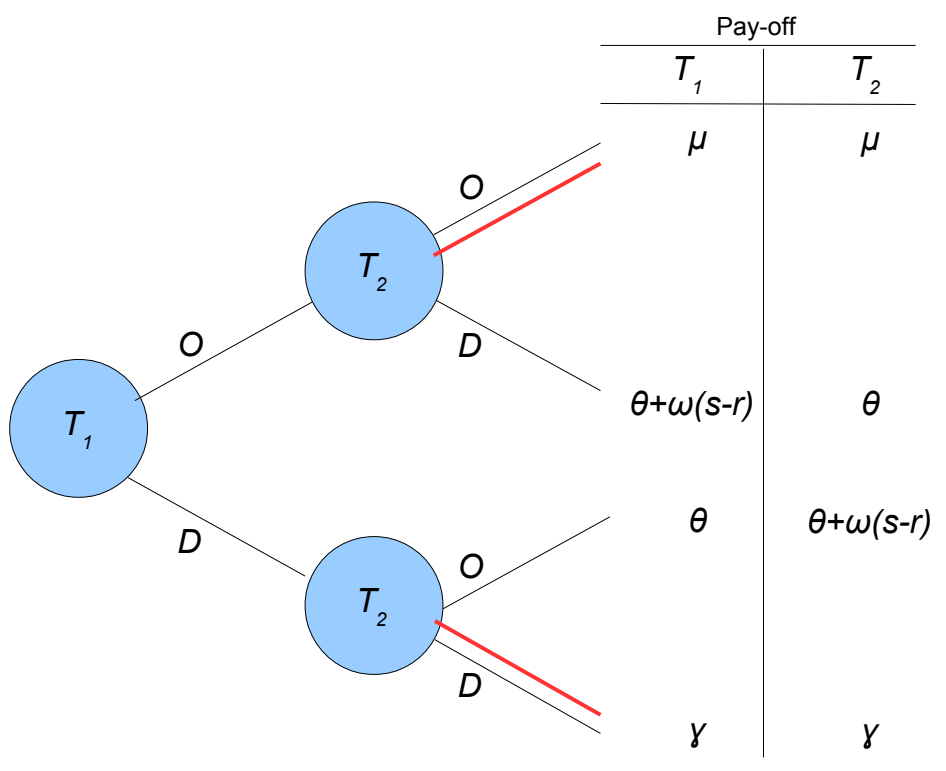

Figure 5. The sequential game with best reply functions for $T_{2}$.

Assumptions (5.1) define only a subspace of possible Nash equilibria in both simultaneous (Figure 1) and sequential games (Figure 4). It will prove interesting to perform a full analysis of the whole $^{4}$ parametric space of these games. The

${ }^{4}$ The cases of equalities (instead of inequalities) are not analysed due to their extreme unlikeliness. 
results (all 4 possible combinations defined by inequalities (4.4) and (4.5)) are summed up by Table $2^{5}$.

\begin{tabular}{c|c|c|c}
$\mu$ & $\gamma$ & Nash eq. & NCNE invariance ${ }^{6}$ \\
\hline$\mu>\theta$ & $\gamma>\theta+\omega(s-r)$ & "Stag-Hunt" & $\mu=\gamma$ \\
$\mu>\theta$ & $\gamma<\theta+\omega(s-r)$ & $\{O O\}$ & Always satisfied \\
$\mu<\theta$ & $\gamma>\theta+\omega(s-r)$ & $\{D D\}$ & Always satisfied \\
$\mu<\theta$ & $\gamma<\theta+\omega(s-r)$ & "Chicken" & $\omega(s-r)=0$
\end{tabular}

Table 2. Analysis of (almost) all situations.

Table 2 contains the relevant different Nash equilibria as well as the necessary conditions for Nash equilibria invariance. The latter concept means a situation in which Nash equilibria are the same in both the simultaneous and the sequential game. As can be observed in Table 2 (with some surprise), both unique pure Nash equilibrium options (the $\{O O\}$ and $\{D D\}$ cases) are always satisfied. This means; given the game set-up, an option of playing a sequential instead of a simultaneous game will never produce different game predictions. The remaining option (last row in Table 2) produces the "Chicken" outcome. That is, 3 Nash equilibria, 2 in pure and one in mixed strategies. This one will only produce NE-invariance if $s=r$. If $s=r$, the team in question plays the $O$ or the $D$ strategy equally well, in practice a highly unlikely situation ${ }^{7}$. Hence, to sum up: One outcome produces incentives to play differently in a sequential game with high probability - the "Chicken" case. The three other cases (given a point score system where $\omega=2 \delta$ ) produce no such incentives to change the behaviour between a simultaneous and a sequential game.

\section{CONClusions AND SUGGestions FOR FURTher WORK}

The most important and interesting finding in this paper is perhaps the important role the structure of the point score system plays in general. Recall the findings in Section 4 and the major impact the simple constraint $\omega=2 \delta$ has on possible game outcomes.

Secondly, the fact that there has been a real-world change from a situation with $\omega=2 \delta$ (the 2-1-0 system) to a situation with $\omega>2 \delta$ (the $3-1-0$ system) indicates, together with the finding leading up to equation (5.2), that the choice of the point score system may have unforeseen consequences. Actually, everything else equal, it is shown here that, for certain parametric combinations (e.g. inequalities (5.1)), there is a greater tendency for secrecy under the 3-1-0 than the 2-1-0 system. As the 3-1-0 system has previously been identified as having negative consequences related to the competitive balance (see [9]), some more questions related to this system's qualities should be asked. Personally, I am in no doubt, reintroduce the original 2-1-0 system.

\footnotetext{
${ }^{5}$ The actual algebra is omitted, but follows the same pattern as demonstrated in the above case. ${ }^{7}$ In fact, this situation could be ruled out at the modelling step as it collapses to a situation where the two strategies are practically equal. It seems sensible to assume that $O$ and $D$ are sufficiently different in such a way that $s \neq r$.
} 
Surely, the analysis presented in this paper is simplified. Two equally good teams are not found in practice, and as such, an added analytic option of unequal team quality seems evident. Although such an added analytic option is both possible and (probably) feasible, this is left for future research.

\section{Appendix A. Proof of Theorem 5.1}

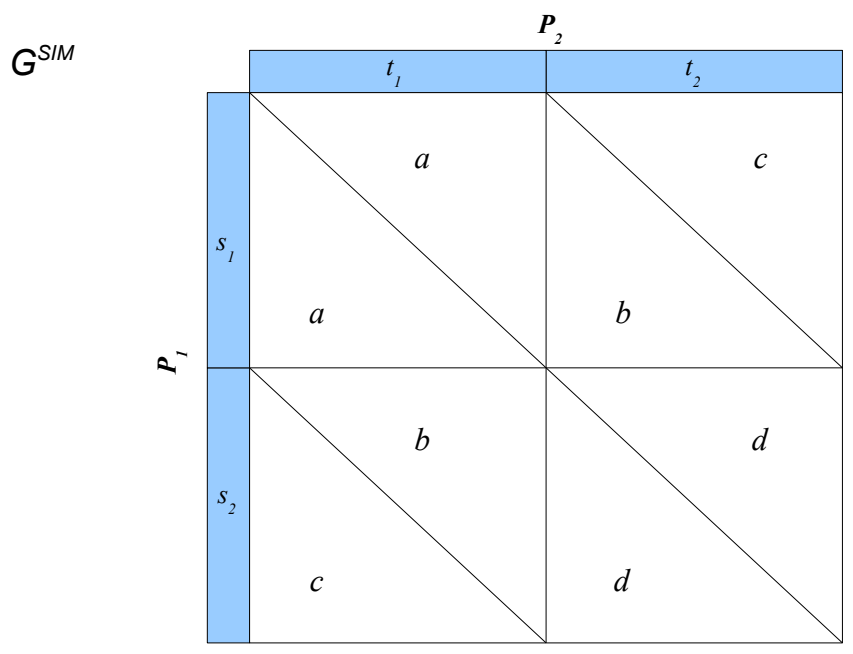

Figure 6. A general simultaneous symmetric 2 by 2 game.

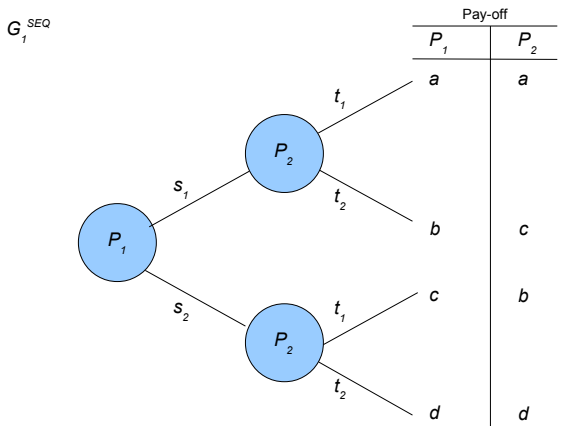

(a) $G_{1}^{S E Q}$
$G_{2}^{S E O}$

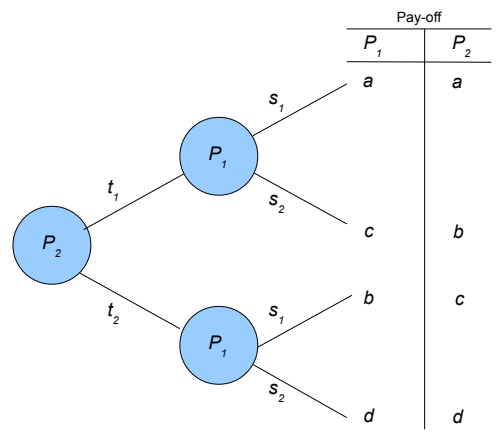

(b) $G_{2}^{S E Q}$

Figure 7. The two possible sequential games based on the simultaneous game.

Proof. Figure 6 contains a general symmetric simultaneous 2 by 2 game named $G^{S I M}$. Figure 7 contains the two possible sequential versions of $G^{S I M}$, named $G_{1}^{S E Q}$ and $G_{2}^{S E Q} . P_{1}$ and $P_{2}$ are players in all games, with strategies $\left\{s_{1}, s_{2}\right\}$ and $\left\{t_{1}, t_{2}\right\}$ for $P_{1}$ and $P_{2}$ respectively. Pay-offs are named $a, b, c, d$. 
Theorem 5.1 states that all possible Nash equilibria in games, $G_{1}^{S E Q}$ and $G_{2}^{S E Q}$ should be the same. This can be proved as follows:

To search for Nash equilibria in sequential games, the Zermelo Algorithm (see for instance [4]) is used. Starting with $G_{1}^{S E Q}$ (left in Figure 7,) a backward induction procedure implies $P_{2}$ solving $\max \{a, c\}$ and $\max \{b, d\}$ at the first stage. As can be readily observed from the right part of Figure $7, P_{1}$ faces exactly the same optimization problems determining his best reply function. Moving to the second stage (the start of the game trees), $P_{1}$ will solve a single optimization problem involving some combination of $\max \{x, y\}$, where $x, y \in\{a, b, x, d\}$. Obviously, a similar situation emerges for $P_{2}$ facing exactly the same optimization problems in $G_{2}^{S E Q}$ as $P_{1}$ did in $G_{1}^{S E Q}$. Consequently, as all optimization problems facing the players are identical, all possible Nash equilibria must be identical.

\section{REFERENCES}

[1] S. Abele and K.-M. Erhardt, The timing effect in public good games, J. Exp. Soc. Psychol. 41 (2005), 470-481.

[2] R. Aoiki and T. J. Prusa, Sequential versus simultaneous choice with endogenous quality, Int. J. Ind. Organ. 15 (1996), 103-121.

[3] K. Bakhrankova, Points scoring systems in Formula 1: A game theoretic perspective, in: D. KLatte, E. Luthi and K. Schmedders (eds.), Operations Research Proceedings 2011, Springer, 2012, pp. 211-216.

[4] K. Binmore, Fun and Games, D.C. Heath and Company, Lexington, Massachusetts, Toronto, 1994.

[5] I. Brochas and J. D. Carillo, Do the "three-point victory" and "golden goal" rules make soccer more exciting?, J. Sports Econ. 5 (2004), 169-185.

[6] S. F. Cheng, D. M. Reeves, Y.Vorobeychik and M. P. Wellman, Notes on equilibria in symmetric games, in: AAMAS-04 Workshop on Game-Theoretic and Decision-Theoretic Agents, New York, 2004, 5 pp.

[7] E. Fernandez-Cantelli and G. Meeden, An improved award system for soccer, Chance 16 (2003), 23-29.

[8] K. K. Haugen, An improved award system for soccer: A (game-theoretic) comment, Chance 20 (2007), 22-24.

[9] K. K. Haugen, Point score systems and competitive balance in professional soccer, J. Sports Econ. 9 (2008), 191-210.

[10] K. K. Haugen, The "Norwegian soccer wonder" - a game theoretic approach, Scandinavian sport studies forum 1 (2010), 1-16.

[11] K. K. Haugen, Always Change a Winning Team, Tapir Academic Publishers, Trondheim, Norway, 2011.

[12] L. Y. Hon and R.A. Parinduri, Does the three-point rule make soccer more exciting? Evidence from a regression discontinuity design, J. Sports Econ. (2014), 19 pp., doi:10.1177/1527002514531790.

[13] M. A. Marini and G. Rodana, Lead, follow or cooperate? Sequential versus collusive payoffs in symmetric duopoly games, ISRN Economics 2013 (2013), 10 pp.

[14] E. Solan and L. Yariv, Games with espionage, Game. Econ. Behav. 47 (2004), 172-199.

Kjetil K. Haugen, Faculty of Logistics, Molde University College, Specialized University in Logistics, Britvegen 2, 6410 Molde, Norway

e-mail: kjetil.haugen@himolde.no 